REPORT

\title{
Biodiversity lessens the risk of cascading extinction in model food webs
}

Charlotte Borrvall, Bo Ebenman and Tomas Jonsson*

Department of Biology,

Linköping University, S-581 83

Linköping, Sweden

E-mail: chbor@ifm.liu.se

*Present address: Laboratory of

Populations, Rockefeller

University, Box 20, 1230 York

Avenue, New York, NY 10021-

6399, USA

\begin{abstract}
Due to the complex interactions between species in food webs, the extinction of one species could lead to a cascade of further extinctions and hence cause dramatic changes in species composition and ecosystem processes. We found that the risk of additional species extinction, following the loss of one species in model food webs, decreases with the number of species per functional group. For a given number of species per functional group, the risk of further extinctions is highest when an autotroph is removed and lowest when a top predator is removed. In addition, stability decreases when the distribution of interaction strengths in the webs is changed from equal to skew (few strong and many weak links). We also found that omnivory appears to stabilize model food webs. Our results indicate that high biodiversity may serve as an insurance against radical ecosystem changes.
\end{abstract}

\section{Keywords}

Biodiversity, extinction, food webs, functional groups, redundancy, resistance, species deletion, stability.

Ecology Letters (2000) 3:131-136

\section{INTRODUCTION}

The relationship between diversity and stability in ecological communities is a long-standing and largely unsettled issue (MacArthur 1955; Elton 1958; May 1972; McNaughton 1977; Cohen \& Newman 1985; Pimm et al. 1991; Tilman 1996; McCann et al. 1998; Tilman 1999) with important implications for the functioning of ecosystems. For instance, theory (Naeem 1998; Yachi \& Loreau 1999), simple laboratory microcosms (McGradySteed et al. 1997; Naeem \& Li 1997) and field experiments of plant communities (Tilman et al. 1996; Tilman et al. 1997) suggest that increased diversity, in the sense of increased number of species per functional group, in ecosystems enhances their functional reliability. The suggested reason for these results is that the failure of one species will be compensated for by other functionally equivalent species. However, due to the complex interactions between species in ecosystems the extinction of one species could lead to additional species losses (cascading extinction) and hence cause dramatic changes in species composition (Paine 1966; Estes \& Palmisano 1974; Mittelbach et al. 1995; Power et al. 1996). For instance, Paine (1966) has shown that predators sometimes enhance species richness through predator-mediated coexistence. According to this mechanism, removal of predators could lead to additional species extinctions. In an early work, MacArthur (1955) argues that predators feeding on many prey are less exposed to variations in prey abundances than predators feeding on fewer prey. Extinction of predators, following the loss of a resource, should therefore decrease with increasing number of prey per predator. Due to the same argument, omnivory should increase food web stability. If the loss of a species causes further extinctions, then the capacity of the ecosystem to remain functionally intact through compensatory growth of other functionally equivalent species might be greatly reduced. An important question is therefore how the risk of cascading extinction events depends on the structure of food webs.

Pioneering studies by Pimm $(1979,1980)$ indicate that complex food webs (webs with many species or high connectance) are more likely to lose additional species following the extinction of one species than simple food webs. The number of species in a food web can be partitioned into two components: number of functional groups and average number of species per functional group. Our analysis differs from Pimm's studies in two important ways. First, keeping the number of functional groups constant we specifically investigate how the degree of redundancy (average number of species per functional group), instead of total species number or connectance, affects the risk of further extinctions following the loss of one species in model food webs. 
Secondly, unlike Pimm (1979, 1980), we also investigate the effects of different distributions of interaction strengths in the webs. In the absence of empirical data theorists have most often assumed distribution of interaction strengths to be uniform or symmetrical (Lawton 1992). However, recent empirical estimates of interaction strengths (e.g. Paine 1992; Wootton 1997) show highly skewed distributions with mostly weak and only a few strong interactions. Using two different distributions, equal and skew, we explore the influence of the distribution of interaction strengths on the risk of losing additional species after the loss of one species. In early descriptions of food webs, omnivory appears to be infrequent (Pimm \& Lawton 1978). By contrast, omnivory seems to be rather common in more recent detailed descriptions of food webs (Sprules \& Bowerman 1988; Polis 1991). In line with this ambiguity we also explore how the presence of omnivory affects the relationship between resistance and redundancy.

Our results suggest that, independent of the distribution of interaction strengths and independent of the presence/absence of omnivory, the risk of cascading extinction events is smaller in webs with many species per functional group than in those with few species per functional group.

\section{METHODS}

\section{Food web model}

We investigate the effects of structural redundancy (average number of species per functional group) on stability (resistance to further extinctions following the loss of one species) using model food webs. In addition we explore how the distribution of interaction strengths and the presence of omnivory affect the relationship between redundancy and stability. The fraction of species in different trophic groups in real food webs is to a large extent unknown and we therefore study two food web geometries: rectangular webs (equal number of species in the different trophic groups) and triangular webs (decreasing number of species with increasing trophic level). For multitrophic systems, functional groups may be operationally defined as trophic groups (Naeem \& Li 1997). Our model food webs consist of three functional/ trophic groups: autotrophs, primary consumers (herbivores) and secondary consumers (potentially omnivorous). The proportion of species in the different functional groups remains constant as the webs increase in size, and the average number of species per functional group spans from two to six. The species in a functional group are ecologically equivalent in the sense of having the same set of prey and predator species (Fig. 1). Thus, species-rich
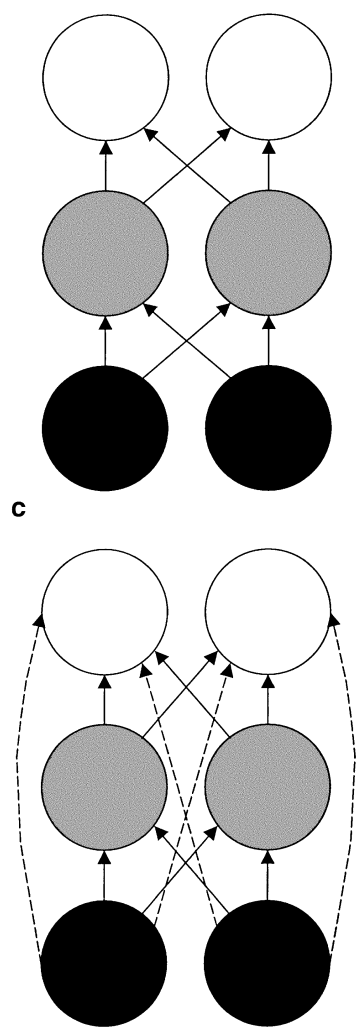
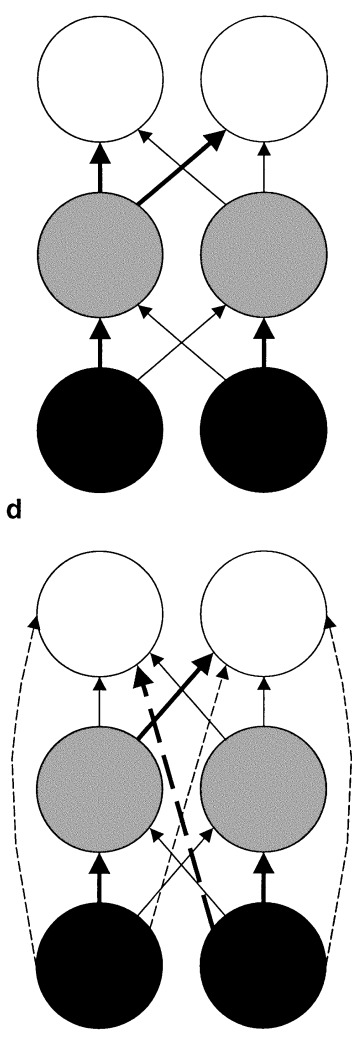

Figure 1 The studied food web configurations (only the four rectangular configurations are shown here) consist of three functional/trophic groups: autotrophs, primary consumers and secondary consumers $(\mathrm{a}, \mathrm{b})$ or omnivores $(\mathrm{c}, \mathrm{d})$. (Solid circles, autotrophs; grey circles, primary consumers; open circles, secondary consumers or omnivores.) Species belonging to the same functional group are trophically equivalent, i.e. they share the same set of prey and predator species. Degree of redundancy (average number of species per functional group) varies from two to six (only the smallest webs are shown here). Each consumer has equal impact on all of its prey (a, c), or each consumer has one strong link (thick line) and many weak links (thin lines) (b, d). The position of the strong link is chosen randomly. Omnivorous links are depicted by dashed lines.

webs have a higher degree of structural redundancy than species-poor ones.

We use the continuous time Lotka-Volterra model:

$$
\frac{\mathrm{d} N_{i}}{\mathrm{~d} t}=N_{i} f_{i}(\boldsymbol{N}) \text { for all } i \in S
$$

where

$$
f_{i}(\boldsymbol{N})=b_{i}+\sum_{j \in S} a_{i j} N_{j}
$$

where $N_{i}$ is the density of species $i, f_{i}$ is its per capita rate of increase, and $\boldsymbol{N}$ is a vector of the densities of all species 
in the set $S$. Autotrophs grow in the absence of consumers $\left(b_{i}>0\right)$ and consumers die in the absence of prey $\left(b_{i}<0\right)$. The effect of species $j$ on species $i$ is given by $a_{i j}$, so that $j$ consumes $i$ when $a_{i j}<0$ and $j$ is prey for $i$ when $a_{i j}>0$. Since the numerical response of a consumer depends on both the number of prey captured per unit time (functional response) and the efficiency with which captured prey are converted into offspring, we model prey effect on consumers as $a_{j i}=-e a_{i j}$, where $e$ is the conversion efficiency with which prey are converted into predators $(e<1$ when the size of the consumer is larger than that of its prey). The strength of direct intraspecific interactions (selflimitation) is given by $a_{i i}<0$. Possible mechanisms behind self-limitation include mutual interference and competition for nonfood resources (Yodzis 1981; Jonsson 1998).

Average per capita effect of a consumer on its prey is assumed to be inversely related to the number of prey it consumes (see McCann et al. 1998). Thus, average effects are weaker in food webs with many species per functional group relative to food webs with few species per functional group. We analyse two possibilities within this scenario: (i) equally distributed interaction strengths. Here, each consumer has equal impact on all of its prey. For this purpose, $a_{i j}$ equals a random number (drawn from the uniform distribution $\left[\begin{array}{ll}-1 & 0\end{array}\right]$ ) divided by the number of prey of that particular predator; (ii) skew distribution of interaction strengths. Here, each predator has a strong effect on one prey (assigned randomly) and weak effects on all others. For this purpose, $a_{i j}$ equals a random number (drawn from the uniform distribution $\left[\begin{array}{ll}-1 & 0\end{array}\right]$ ) times 0.9 (for the strong link) or times 0.1 divided by the number of prey minus one (for the weak links). For each species the strength of intraspecific interaction $\left(a_{i i}\right)$ is randomly drawn from the uniform distribution $\left[\begin{array}{ll}-1 & 0\end{array}\right]$. In order to reduce simulation time (increase number of locally stable replicates), mean intraspecific interactions are kept stronger relative to predator interactions. This does not affect the overall trend in our results. Mortality rates for the consumers $\left(b_{j}\right)$ are randomly drawn from the uniform distribution $[-0.010]$. The mortality rates are then sorted in such a way that the secondary consumers are assigned lower rates than their prey (herbivores) because species occupying higher trophic levels are expected to be larger (Cohen et al. 1993; Jonsson 1998) and a large size often confers low mortality rate (Calder 1984). The intervals for the parameters in the present study are similar to the ones used and motivated in earlier theoretical studies of food webs (e.g. Pimm 1979; Pimm 1980).

In summary we have eight food web configurations (see Fig. 1) differing from each other concerning geometry (rectangular/triangular), distribution of interaction strengths (equal/skew) and omnivory (presence/ absence). For each food web in these eight configurations we produce 10000 replicates in which the parameters are sampled from the above distributions. We remove one species from those replicates having a feasible (all species densities are positive) and locally stable equilibrium. The community is termed resistant if the reduced community (after the removal of one species) remains feasible and locally stable, implying that no further species have been lost. The analysis is repeated removing each species in the web but only one in each analysis.

Systems may exist although they do not move towards an equilibrium point, meaning that the criterion of local stability might miss some persistent systems (Hutson \& Schmitt 1992; Law \& Morton 1996). However, in our analyses all locally unstable systems were also unfeasible. (One possible explanation for this might be an absence of complex dynamics due to all species having intraspecific density dependence and to the absence of a saturating functional response.) Hence, local instability tells us that one or more species will be lost as a consequence of the removal of one species.

\section{RESULTS}

We find an overall trend of increasing food web resistance with increasing number of species per functional group (Figs 2, 3). Adding species to the functional groups makes the food web more resistant to further extinction following the loss of one species, indicating that trophically equivalent species enhance food web stability. This positive diversity-stability trend occurs both with (Fig. 2c, d, 3c, d) and without omnivory (Fig. 2a, b, 3a, b) and whether consumers affect all of their prey equally (Fig. 2a, c, 3a, c) or each consumer has a large impact on one of its prey but small impact on the rest (Fig. 2b, d, 3b, d). Moreover, the trend is the same both for rectangular (Fig. 2) and triangular webs (Fig. 3). However, the risk of losing additional species is consistently higher in rectangular food webs compared with triangular ones, particularly when an autotroph is lost.

For a given number of species per functional group, resistance is highest when a top predator is lost, intermediate when a herbivore is lost and lowest when an autotroph is lost (Figs 2, 3). An exception from this is triangular webs without omnivory (Fig. 3a, b). Here, resistance is somewhat higher when an autotroph is lost compared with when a herbivore is lost. The probability of further species losses is almost zero when a top predator is removed.

When a top predator or omnivore is removed, resistance is independent of the way in which the interaction strengths are distributed. However, when a herbivore or an autotroph is removed, resistance is markedly higher when the consumer affects all of its prey 

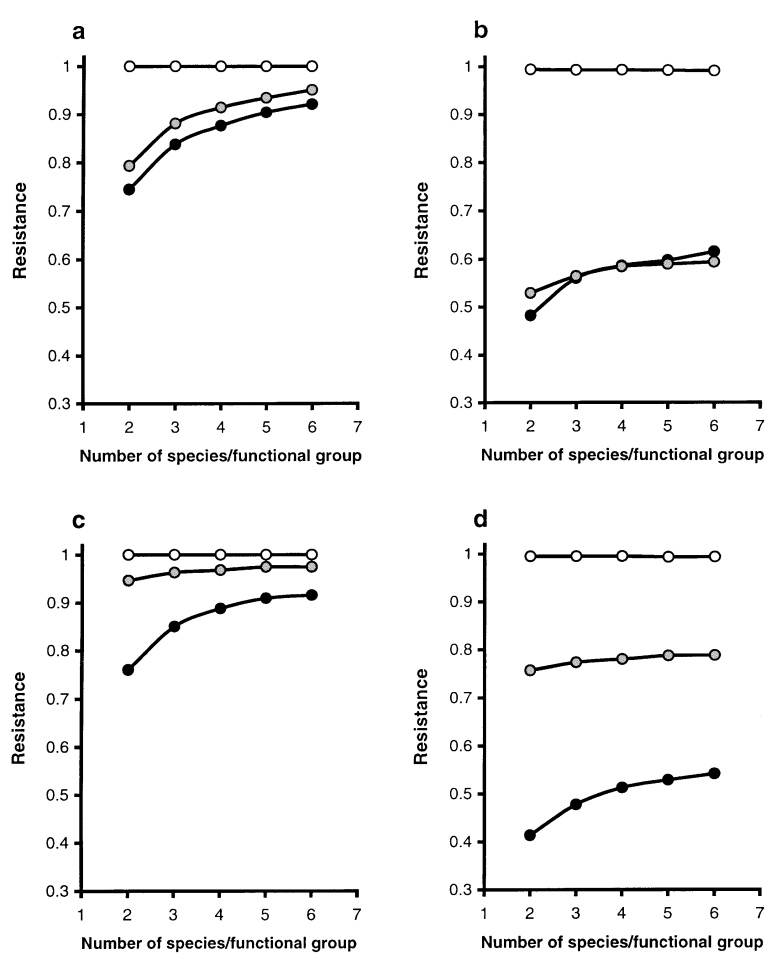

Figure 2 Resistance against further species extinction events following the removal of one species (measured as the probability to remain locally stable after the removal of one species) as a function of redundancy (measured as the number of species per functional group in the food web) in rectangular food webs. The four corresponding food web configurations are given in Fig. 1. (a) No omnivory, equal interaction strengths; (b) no omnivory, skew interaction strengths; (c) omnivory included, equal interaction strengths; (d) omnivory included, skew interaction strengths. Parameter values: $b i=1$ for autotrophs; $e=0.1$ for ordinary links and $e=0.01$ for omnivorous links; other parameters are sampled from predefined intervals. Each curve corresponds to the removal of species from one of the functional groups. Solid circles, removal of autotrophs; grey circles, removal of primary consumers; open circles, removal of secondary consumers or omnivores. For the system with four species per functional group the number of feasible and locally stable replicates was 4048 with no omnivory and equal interaction strengths, 4385 with no omnivory and skew interaction strengths, 4972 with omnivory and equal interaction strengths and 4964 with omnivory and skew interaction strengths. With fewer species per functional group a larger fraction of the 10000 replicates was feasible and locally stable and with more species per functional group a smaller fraction was feasible and locally stable.

equally (Fig. 2a, c, 3a, c), compared with when it has strong impact on one of its prey but weak impact on the others (Fig. 2b, d, 3b, d).

The major effect of omnivory is to lessen the risk of further species extinctions following the loss of a herbivore.
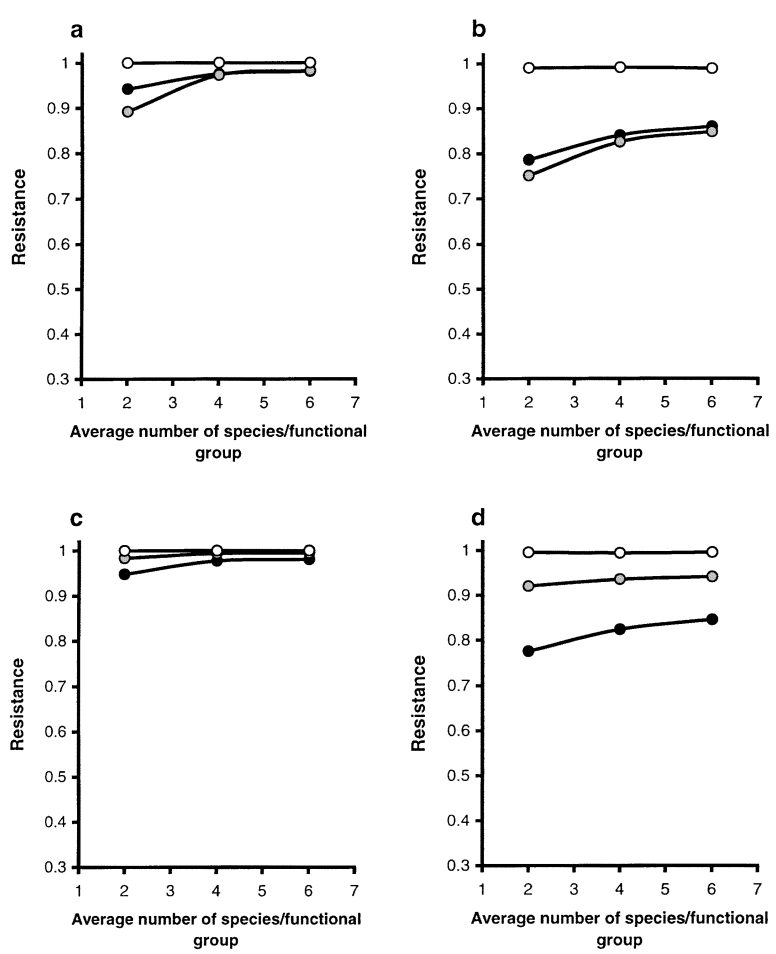

Figure 3 Resistance against further species extinction events following the removal of one species (measured as the probability to remain locally stable after the removal of one species) as a function of redundancy (measured as the average number of species per functional group in the food web) in triangular food webs. For food web configurations and parameter values, see legend of Fig. 2. For the system with an average of four species per functional group the number of feasible and locally stable replicates was 6632 with no omnivory and equal interaction strengths, 7121 with no omnivory and skew interaction strengths, 7620 with omnivory and equal interaction strengths and 7594 with omnivory and skew interaction strengths. With fewer species per functional group a larger fraction of the 10000 replicates was feasible and locally stable and with more species per functional group a smaller fraction was feasible and locally stable.

\section{DISCUSSION}

According to our results, food webs with many species per functional group may be more resistant to species loss than webs with few species per functional group, in the sense that the risk of further species extinction following the loss of one species is lower in species-rich webs than in species-poor ones. This lends additional support to the hypothesis (Walker 1992; Naeem 1998) and empirical result (McGrady-Steed et al. 1997) that high biodiversity can be seen as an insurance against radical ecosystem changes even though species may have largely redundant functional roles. The result seems rather robust in that the overall trend of increasing resistance with increasing structural redundancy is the same for rectangular and 
triangular food webs, and it also remains when omnivory is included in the webs and when the distribution of interaction strengths is changed from equal to skew. In the present analysis, predator effects are assumed to decrease with increasing number of prey per predator. It might therefore be argued that the trend of increasing stability with increasing diversity is caused by decreased per capita effects and not by diversity per se. However, relaxation of this assumption does not alter the diversitystability trend found, making our result even more robust.

The probability of further species losses is almost zero when a top predator is removed. A possible explanation for this lack of predator-mediated coexistence is the absence of interspecific interference competition between primary consumers (herbivores) in our model. This makes it less likely that any herbivore species will dominate and eliminate the others in the absence of predators (e.g. Paine 1966). Thus, the loss of predators may not lead to additional losses of herbivore species. Moreover, the presence of intraspecific interference competition in the herbivores in our model might make it less likely that they will overgraze and eliminate autotrophs in the absence of predators (e.g. Estes \& Palmisano 1974). Even though there are some fascinating examples of predator-mediated coexistence (e.g. Paine 1966; Lubchenco 1978) it may not be a very common phenomenon in natural communities (for a review see Morin 1999). In fact, top predators may more often have the opposite effect: reducing diversity and creating communities dominated by species most resistant to predation (Morin 1999). It is also interesting to note that in line with the theoretical results found here, Sih et al. (1985), reviewing data on experimental manipulation of predators, found that striking effects of consumers on species composition were most common for consumers feeding lower in the food chain.

When a primary producer or a herbivore is lost our results are in line with Pimm's findings (1980): the risk of losing additional species decreases with increasing number of prey species in the diet of consumers. However, when a top predator is removed, Pimm found decreasing stability with increasing number of herbivores in the predators' diet and increasing number of plant species consumed by these herbivores, which is in contrast to our result. The most likely explanation for this difference is the presence of intraspecific interference competition in consumers in our model. As mentioned above, the presence of intraspecific interference competition in herbivores might decrease the risk of species losses within the lowest trophic level following the removal of a top predator. It is also worth pointing out that Pimm varied the degree of connectance of the food webs (keeping the number of species constant) while we vary the degree of redundancy (number of species per functional group, keeping connectance almost constant).
For a given number of species per functional group, webs with equally distributed interaction strengths show higher resistance compared with webs containing both strong and weak interactions. In those webs having skew interactions, most of the links are weak. This means that most of the links being lost when a species is removed are weak. Consequently, our result indicates that removal of weak links, in the presence of strong ones, can destabilize food webs. This is consistent with the recent finding that addition of weak links can stabilize the dynamics of simple strongly coupled food webs by dampening oscillations between consumers and resources (McCann et al. 1998).

It has been suggested, using Lotka-Volterra models of food webs, that the presence of omnivory destabilizes ecological communities (Pimm \& Lawton 1978). However, recent theoretical studies, adopting a nonequilibrium perspective, show that the addition of omnivorous links to simple three-species food chain models has a stabilizing effect in that oscillations tend to be eliminated or damped (McCann \& Hastings 1997). Using the resistance to further species extinctions as the criterion of stability we also find a stabilizing effect of omnivory when a herbivore is lost. Thus, when a herbivore species is lost from the food web, resistance is higher in webs with omnivory than in webs without omnivorous links. In line with MacArthur's (1955) early suggestions concerning decreased variability with increased number of prey per predator, this seems reasonable as those top predators feeding on the lost herbivore have more alternative prey when omnivory is present.

The geometry of food webs also affects their stability. For a given number of species, triangular webs are more resistant to further species extinctions than rectangular webs following the loss of an autotroph or a herbivore. The difference is most pronounced when an autotroph is lost. One likely explanation for this difference is that, for a given number of species, triangular webs have a higher degree of redundancy in the autotroph functional group than rectangular webs. This means that there is more alternative prey for herbivores in triangular webs than in rectangular ones. Hence, the loss of an autotroph should have a smaller impact on herbivores in triangular webs compared with rectangular webs. Also this result is consistent with MacArthur's (1955) theory.

In this study we have deleted all species, one at a time; but which species in the food webs are most vulnerable to extinction in the first place? Some studies indicate that extinctions following habitat fragmentation are disproportionately experienced by species at high trophic levels (discussed by Holt et al. 1999). Although species at higher trophic levels might be more prone to extinction than species at lower trophic levels, this study indicates that the loss of species from lower trophic levels causes greater risks of losing additional species. Thus, when it comes to conservational efforts our results show that it might not 
be enough only to care about those species tending to go extinct in the first place but also take into account what happens as a consequence of the loss of other less extinction-prone species.

\section{ACKNOWLEDGEMENTS}

We thank J.E. Cohen and R. Law for discussion and valuable comments; three anonymous reviewers for valid criticisms and advice; and The Royal Swedish Academy of Science (Crafoord Foundation), the Swedish Council for Planning and Coordination of Research, and the Foundation for Strategic Research for their support.

\section{REFERENCES}

Calder, W.A. III (1984). Size, Function, and Life History. Harvard University Press, Cambridge.

Cohen, J.E. \& Newman, C.M. (1985). When will a large complex system be stable? J. Theoret. Biol., 113, 153-156.

Cohen, J.E., Pimm, S.L., Yodzis, P. \& Saldaña, J. (1993). Body sizes of animal predators and animal prey in food webs. $J$. Anim. Ecol., 62, 67-78.

Elton, C.S. (1958). The Ecology of Invasions by Animals and Plants. Chapman \& Hall, London.

Estes, J.A. \& Palmisano, J.F. (1974). Sea otters: their role in structuring nearshore communities. Science, 185, 1058-1060.

Holt, R.D., Lawton, J.H., Polis, G.A. \& Martinez, N.D. (1999). Trophic rank and the species-area relationship. Ecology, 80, 1495-1504.

Hutson, V. \& Schmitt, K. (1992). Permanence and the dynamics of biological systems. Math. Bioscience, 111, 1-71.

Jonsson, T. (1998). Food webs and the distribution of body sizes. Linköping Studies in Science and Technology.Dissertation, 535, Linköping.

Law, R. \& Morton, D.R. (1996). Permanence and the assembly of ecological communities. Ecology, 77, 762-775.

Lawton, J.H. (1992). Feeable links in food webs. Nature, 355, 19-20.

Lubchenco, J. (1978). Plant species diversity in a marine intertidal community: importance of herbivore food preferences and algal competitive abilities. Am. Naturalist, 112, 23-39.

MacArthur, R.H. (1955). Fluctuations of animal populations, and a measure of community stability. Ecology, 36, 533-536.

May, R. (1972). Will a large complex system be stable? Nature, 238, 413-414.

McCann, K. \& Hastings, A. (1997). Re-evaluating the omnivory-stability relationship in food webs. Proc. R. Soc. London. Series B, 264, 1249-1254.

McCann, K., Hastings, A. \& Huxel, G.R. (1998). Weak trophic interactions and the balance of nature. Nature, 395, 794-798.

McGrady-Steed, J., Harris, P.M. \& Morin, P. J. (1997). Biodiversity regulates ecosystem predictability. Nature, 390, 162-164.

McNaughton, S.J. (1977). Diversity and stability of ecological communities: a comment on the role of empiricism in ecology. Am. Naturalist, 111, 515-525.

Mittelbach, G.G., Turner, A.M., Hall, D.J. \& Rettig, J.E. (1995). Perturbation and resilience: a long-term, whole-lake study of predator extinction and reintroduction. Ecology, 76, 2347-2360.

Morin, P. J. (1999). Community Ecology. Blackwell Science, Boston.
Naeem, S. (1998). Species redundancy and ecosystem reliability. Conservation Biol., 12, 39-45.

Naeem, S. \& Li, S. (1997). Biodiversity enhances ecosystem reliability. Nature, 390, 507-509.

Paine, R.T. (1966). Food web complexity and species diversity. Am. Naturalist, 100, 65-75.

Paine, R.T. (1992). Food-web analysis through field measurement of per capita interaction strength. Nature, 355, 73-75.

Pimm, S.L. (1979). Complexity and stability: another look at MacArthur's original hypothesis. Oikos, 33, 351-357.

Pimm, S.L. (1980). Food web design and the effect of species deletion. Oikos, 35, 139-149.

Pimm, S.L. \& Lawton, J.H. (1978). On feeding on more than one trophic level. Nature, 275, 542-544.

Pimm, S.L., Lawton, J.H. \& Cohen, J.E. (1991). Food web patterns and their consequences. Nature, 350, 669-674.

Polis, G.A. (1991). Complex desert food webs: an empirical critique of food web theory. Am. Naturalist, 138, 123-155.

Power, M.E., Tilman, D., Estes, J.A., Menge, B.A., Bond, W. J., Mills, L.S., Daily, G., Castilla, J., Lubchenco, J. \& Paine, R.T. (1996). Challenges in the quest for keystones. Bioscience, 46, 609-620.

Sih, A., Crowley, P., McPeek, M., Petranka, J. \& Strohmeier, K. (1985). Predation, competition, and prey communities: a review of field experiments. Annu. Rev. Ecol. Syst, 16, 269-311.

Sprules, W.G. \& Bowerman, J.E. (1988). Omnivory and food chain length in zooplankton food webs. Ecology, 69, 418-426.

Tilman, D. (1996). Biodiversity: population versus ecosystem stability. Ecology, 77, 350-363.

Tilman, D. (1999). The ecological consequences of changes in biodiversity: a search for general principles. Ecology, 80, 1455-1474.

Tilman, D., Wedin, D. \& Knops, J. (1996). Productivity and sustainability influenced by biodiversity in grassland ecosystems. Nature, 379, 718-720.

Tilman, D., Knops, J., Wedin, D., Reich, P., Ritchie, M. \& Siemann, E. (1997). The influence of functional diversity and composition on ecosystem processes. Science, 277, 1300-1302.

Walker, B. (1992). Biodiversity and ecological redundancy. Conservation Biol., 6, 18-23.

Wootton, J.T. (1997). Estimates and tests of per capita interaction strength: diet, abundance, and impact of intertidally foraging birds. Ecol. Monographs, 67, 45-64.

Yachi, S. \& Loreau, M. (1999). Biodiversity and ecosystem productivity in a fluctuating environment: The insurance hypothesis. Proc. Natl. Acad. Sci., 96, 1463-1468.

Yodzis, P. (1981). The stability of real ecosystems. Nature, 289, 674-676.

\section{BIOSKETCH}

Charlotte Borrvall is interested in theoretical ecology, particularly stability and complexity of food webs. She likes programming and thinks that ecology gains a lot from computer simulations and mathematical models.

Editor, M. Loreau

Manuscript received 4 October 1999

First decision made 15 November 1999

Manuscript accepted 6 January 2000 